\title{
Ranks and Kernels of Codes from Generalized Hadamard Matrices
}

\author{
Steven T. Dougherty, Josep Rifà, Senior Member, IEEE, Mercè Villanueva
}

\begin{abstract}
The ranks and kernels of generalized Hadamard matrices are studied. It is proven that any generalized Hadamard matrix $H(q, \lambda)$ over $\mathbb{F}_{q}, q>3$, or $q=3$ and $\operatorname{gcd}(3, \lambda) \neq 1$, generates a self-orthogonal code. This result puts a natural upper bound on the rank of the generalized Hadamard matrices. Lower and upper bounds are given for the dimension of the kernel of the corresponding generalized Hadamard codes. For specific ranks and dimensions of the kernel within these bounds, generalized Hadamard codes are constructed.
\end{abstract}

Index Terms-Generalized Hadamard matrix, generalized Hadamard code, rank, kernel, nonlinear code, self-orthogonal code

\section{INTRODUCTION}

Let $\mathbb{F}_{q}=\mathrm{GF}(q)$ denote the finite field with $q$ elements, where $q=p^{e}, p$ prime. Let $\mathbb{F}_{q}^{n}$ be the vector space of dimension $n$ over $\mathbb{F}_{q}$. The Hamming distance between vectors $\mathbf{w}, \mathbf{v} \in \mathbb{F}_{q}^{n}$, denoted by $d(\mathbf{w}, \mathbf{v})$, is the number of coordinates in which $\mathbf{w}$ and $\mathbf{v}$ differ. A code $C$ over $\mathbb{F}_{q}$ of length $n$ is a nonempty subset of $\mathbb{F}_{q}^{n}$. The elements of $C$ are called codewords. A code $C$ over $\mathbb{F}_{q}$ is called linear if it is a linear space over $\mathbb{F}_{q}$ and, it is called $K$-additive if it is a linear space over a subfield $K \subset \mathbb{F}_{q}$. The dimension of a $K$-additive code $C$ over $\mathbb{F}_{q}$ is defined as the number $k$ such that $q^{k}=|C|$. Note that $k$ is not necessarily an integer, but $k e$ is an integer, where $q=|K|^{e}$. The minimum distance of a code is the smallest Hamming distance between any pair of distinct codewords. Two codes $C_{1}, C_{2} \subset \mathbb{F}_{q}^{n}$ are said to be permutation equivalent if there exists a permutation $\sigma$ of the $n$ coordinates such that $C_{2}=\left\{\sigma\left(c_{1}, c_{2}, \ldots, c_{n}\right)=\right.$ $\left.\left(c_{\sigma^{-1}(1)}, \ldots, c_{\sigma^{-1}(n)}\right):\left(c_{1}, c_{2}, \ldots, c_{n}\right) \in C_{1}\right\}$, [3], [10]. Without loss of generality, we shall assume, unless stated otherwise, that the all-zero vector, denoted by $\mathbf{0}$, is in $C$.

Two structural parameters of (nonlinear) codes are the dimension of the linear span and the kernel. The linear span of a code $C$ over $\mathbb{F}_{q}$, denoted by $\mathcal{R}(C)$, is the subspace over $\mathbb{F}_{q}$ spanned by $C$, that is $\mathcal{R}(C)=\langle C\rangle$. The dimension of $\mathcal{R}(C)$ is called the rank of $C$ and is denoted by $\operatorname{rank}(C)$. If $q=p^{e}, p$ prime, we can also define $\mathcal{R}_{p}(C)$ and $\operatorname{rank}_{p}(C)$ as the subspace over $\mathbb{F}_{p}$ spanned by $C$ and its dimension,

This work was partially supported by the Spanish MICINN under Grant TIN2013-40524-P, and by the Catalan AGAUR under Grant 2014SGR-691.

Steven T. Dougherty is with the Department of Mathematics, University of Scranton, Scranton PA 18510, USA.

Josep Rifà and Mercè Villanueva are with the Department of Information and Communications Engineering, Universitat Autònoma de Barcelona, 08193 Cerdanyola del Vallès, Spain. respectively. The kernel of a code $C$ over $\mathbb{F}_{q}$, denoted by $\mathcal{K}(C)$, is defined as $\mathcal{K}(C)=\left\{\mathbf{x} \in \mathbb{F}_{q}^{n}: \alpha \mathbf{x}+C=C\right.$ for all $\left.\alpha \in \mathbb{F}_{q}\right\}$. If $q=p^{e}, p$ prime, we can also define the p-kernel of $C$ as $\mathcal{K}_{p}(C)=\left\{\mathbf{x} \in \mathbb{F}_{q}^{n}: \mathbf{x}+C=C\right\}$. Since we assume that $\mathbf{0} \in C$, then $\mathcal{K}(C)$ is a linear subcode of $C$ and $\mathcal{K}_{p}(C)$ is an $\mathbb{F}_{p}$-additive subcode. We denote the dimension of the kernel and $p$-kernel of $C$ by $\operatorname{ker}(C)$ and $\operatorname{ker}_{p}(C)$, respectively. These concepts were first defined in $[15]$ for codes over $\mathbb{F}_{q}$, generalizing the binary case described previously in [2], [14]. In [15], it was proved that the code $C$ over $\mathbb{F}_{q}$ can be written as the union of cosets of $\mathcal{K}(C)$ (resp. $\left.\mathcal{K}_{p}(C)\right)$, and $\mathcal{K}(C)$ (resp. $\mathcal{K}_{p}(C)$ ) is the largest such linear code over $\mathbb{F}_{q}$ (resp. $\mathbb{F}_{p}$ ) for which this is true. Moreover, it is clear that $\mathcal{K}(C) \subseteq \mathcal{K}_{p}(C)$.

A generalized Hadamard $(\mathrm{GH})$ matrix $H(q, \lambda)=\left(h_{i j}\right)$ of order $n=q \lambda$ over $\mathbb{F}_{q}$ is a $q \lambda \times q \lambda$ matrix with entries from $\mathbb{F}_{q}$ with the property that for every $i, j, 1 \leq i<j \leq q \lambda$, each of the multisets $\left\{h_{i s}-h_{j s}: 1 \leq s \leq q \lambda\right\}$ contains every element of $\mathbb{F}_{q}$ exactly $\lambda$ times. It is known that since $\left(\mathbb{F}_{q},+\right)$ is an abelian group then $H(q, \lambda)^{T}$ is also a $\mathrm{GH}$ matrix, where $H(q, \lambda)^{T}$ denotes the transpose of $H(q, \lambda)$ [9]. An ordinary Hadamard matrix of order $4 \mu$ corresponds to a GH matrix $H(2, \lambda)$ over $\mathbb{F}_{2}$, where $\lambda=2 \mu$.

Two GH matrices $H_{1}$ and $H_{2}$ of order $n$ are said to be equivalent if one can be obtained from the other by a permutation of the rows and columns and adding the same element of $\mathbb{F}_{q}$ to all the coordinates in a row or in a column. We can always change the first row and column of a GH matrix into zeros and we obtain an equivalent GH matrix which is called normalized. From a normalized Hadamard matrix $H$, we denote by $F_{H}$ the code over $\mathbb{F}_{q}$ consisting of the rows of $H$, and $C_{H}$ the one defined as $C_{H}=\bigcup_{\alpha \in \mathbb{F}_{q}}\left(F_{H}+\alpha \mathbf{1}\right)$, where $F_{H}+\alpha \mathbf{1}=\{\mathbf{h}+\alpha \mathbf{1}: \mathbf{h} \in$ $\left.F_{H}\right\}$ and $\mathbf{1}$ denotes the all-one vector. The code $C_{H}$ over $\mathbb{F}_{q}$ is called generalized Hadamard $(\mathrm{GH})$ code. Note that $F_{H}$ and $C_{H}$ are generally nonlinear codes over $\mathbb{F}_{q}$.

To check whether two GH matrices are equivalent is known to be an NP-hard problem. However, we can use the invariants related to the linear span and kernel of the corresponding GH codes in order to help in their classification, since if two GH codes have different ranks or dimensions of the kernel, the GH matrices are nonequivalent.

Lemma 1.1: Let $H$ be a GH matrix over $\mathbb{F}_{q}$. Then $\operatorname{rank}\left(C_{H}\right)=\operatorname{rank}\left(F_{H}\right)+1$ and $\operatorname{ker}\left(C_{H}\right)=\operatorname{ker}\left(F_{H}\right)+1$.

Proof: It is straightforward from the definitions. 
In this paper, we shall only study GH codes over a finite field because of the following proposition.

Proposition 1.2: Let $H$ be a GH matrix over a ring $R$ that is not a field. Then $\mathcal{K}\left(F_{H}\right)$ is trivial.

Proof: If $\mathbf{v}$ is a row of $H$ and $\mathbf{v} \in \mathcal{K}\left(F_{H}\right)$, then $\alpha \mathbf{v}$ must be in $F_{H}$ for all $\alpha$ in the ring. If $\alpha$ is a non-unit, $\alpha \mathbf{v}$ cannot have, as coordinates, every element of $R \lambda$ times and so $\alpha \mathbf{v}$ is not in $F_{H}$. Hence the kernel is trivial.

The rank and dimension of the kernel for ordinary Hadamard codes over $\mathbb{F}_{2}$ have been studied in [16], [17], [18]. Specifically, lower and upper bounds for these two parameters were established, and the construction of an Hadamard code for all allowable ranks and dimensions of the kernel between these bounds was also given.

In this paper, we present a generalization of the above results. The paper is organized as follows. In Section II, we give the implications for the rank and kernel for the standard Kronecker sum construction. In Section III, we find lower and upper bounds for the dimension of the kernel of a GH code, constructing examples for some specific values in this interval. In Section IV, we establish an upper bound for the rank, by proving that the GH codes are self-orthogonal unless $q=3$ and $\operatorname{gcd}(\lambda, q)=1$. Finally, in Section $\mathrm{V}$, we give some conclusions and discuss further avenues of research on this topic.

\section{KRONECKER SUM CONSTRUCTION}

A standard method to construct $\mathrm{GH}$ matrices from other GH matrices is given by the Kronecker sum construction [13], [22]. That is, if $H(q, \lambda)=\left(h_{i j}\right)$ is any $q \lambda \times q \lambda \mathrm{GH}$ matrix over $\mathbb{F}_{q}$, and $B_{1}, B_{2}, \ldots, B_{q \lambda}$ are any $q \mu \times q \mu \mathrm{GH}$ matrices over $\mathbb{F}_{q}$, then the matrix in Table I gives a $q^{2} \lambda \mu \times q^{2} \lambda \mu \mathrm{GH}$ matrix over $\mathbb{F}_{q}$, denoted by $H \oplus\left[B_{1}, B_{2}, \ldots, B_{n}\right]$, where $n=q \lambda$. If $B_{1}=B_{2}=\cdots=$ $B_{n}=B$, then we write $H \oplus\left[B_{1}, B_{2}, \ldots, B_{n}\right]=H \oplus B$.

TABLE I

KRONECKER SUM CONSTRUCTION

$$
\left(\begin{array}{cccc}
H \oplus\left[B_{1}, B_{2}, \ldots, B_{n}\right]= \\
h_{11}+B_{1} & h_{12}+B_{1} & \cdots & h_{1 n}+B_{1} \\
h_{21}+B_{2} & h_{22}+B_{2} & \cdots & h_{2 n}+B_{2} \\
\vdots & \vdots & \vdots & \vdots \\
h_{n 1}+B_{n} & h_{n 2}+B_{n} & \cdots & h_{n n}+B_{n}
\end{array}\right)
$$

Let $S_{q}$ be the normalized GH matrix $H(q, 1)$ given by the multiplicative table of $\mathbb{F}_{q}$. As for ordinary Hadamard matrices over $\mathbb{F}_{2}$, starting from a GH matrix $S^{1}=S_{q}$, we can recursively define $S^{t}$ as a GH matrix $H\left(q, q^{t-1}\right)$, constructed as $S^{t}=S_{q} \oplus\left[S^{t-1}, S^{t-1}, \ldots, S^{t-1}\right]=S_{q} \oplus S^{t-1}$ for $t>1$, which is called a Sylvester GH matrix.

Let $\mathbf{v}=\left(v_{1}, v_{2}, \ldots, v_{n}\right)$ and $\mathbf{w}=\left(w_{1}, w_{2}, \ldots, w_{m}\right)$ be two vectors over $\mathbb{F}_{q}$. Then, $\mathbf{v} \oplus \mathbf{w}=\left(v_{1}+w_{1}, \ldots, v_{1}+\right.$ $\left.w_{m}, v_{2}+w_{1}, \ldots, v_{2}+w_{m}, \ldots, v_{n}+w_{1}, \ldots, v_{n}+w_{m}\right)$.

Lemma 2.1: Let $H_{1}$ and $H_{2}$ be two GH matrices over $\mathbb{F}_{q}$ and $H=H_{1} \oplus H_{2}$. Then $\operatorname{rank}\left(C_{H}\right)=\operatorname{rank}\left(C_{H_{1}}\right)+$ $\operatorname{rank}\left(C_{H_{2}}\right)-1$ and $\operatorname{ker}\left(C_{H}\right)=\operatorname{ker}\left(C_{H_{1}}\right)+\operatorname{ker}\left(C_{H_{2}}\right)-1$.

Proof: Without loss of generality, assume that $H_{1}(q, \lambda)$ and $H_{2}(q, \mu)$ are normalized $\mathrm{GH}$ matrices with row vectors $\mathbf{v}_{i} \in H_{1}$ and $\mathbf{w}_{j} \in H_{2}, i \in\{1, \ldots, q \lambda\}$ and $j \in\{1, \ldots, q \mu\}$. Since $\mathbf{v}_{i} \oplus \mathbf{w}_{j}=\mathbf{v}_{i} \oplus \mathbf{0}+\mathbf{0} \oplus \mathbf{w}_{j}$, it is easy to see that all row vectors in $H=H_{1} \oplus H_{2}$ are linearly generated by $\mathbf{v}_{i} \oplus \mathbf{0}$ and $\mathbf{0} \oplus \mathbf{w}_{j}$. Moreover, since the two vectors $\mathbf{v}_{i} \oplus \mathbf{0}$ and $\mathbf{0} \oplus \mathbf{w}_{j}$ are linearly independent for any $\mathbf{v}_{i} \neq \mathbf{0}$ and $\mathbf{w}_{j} \neq \mathbf{0}$, we have that $\operatorname{rank}\left(F_{H}\right)=\operatorname{rank}\left(F_{H_{1}}\right)+\operatorname{rank}\left(F_{H_{2}}\right)$. By Lemma 1.1, $\operatorname{rank}\left(C_{H}\right)=\operatorname{rank}\left(F_{H}\right)+1=\operatorname{rank}\left(F_{H_{1}}\right)+\operatorname{rank}\left(F_{H_{2}}\right)+1=$ $\operatorname{rank}\left(C_{H_{1}}\right)+\operatorname{rank}\left(C_{H_{2}}\right)-1$.

For the kernel, we have that $\mathbf{v}_{i} \oplus \mathbf{w}_{j} \in \mathcal{K}\left(F_{H}\right)$ if and only if $\mathbf{v}_{i} \in \mathcal{K}\left(F_{H_{1}}\right)$ and $\mathbf{w}_{j} \in \mathcal{K}\left(F_{H_{2}}\right)$. Hence, the vectors $\mathbf{v}_{i} \oplus \mathbf{0}$ and $\mathbf{0} \oplus \mathbf{w}_{j}$, where $\mathbf{v}_{i} \in \mathcal{K}\left(F_{H_{1}}\right)$ and $\mathbf{w}_{j} \in \mathcal{K}\left(F_{H_{2}}\right)$, linearly generate $\mathcal{K}\left(F_{H}\right)$ and so, $\operatorname{ker}\left(F_{H}\right)=\operatorname{ker}\left(F_{H_{1}}\right)+\operatorname{ker}\left(F_{H_{2}}\right)$. Finally, the result follows by Lemma 1.1.

Corollary 2.2: Let $B$ be a $\mathrm{GH}$ matrix over $\mathbb{F}_{q}$ and $H=$ $S_{q} \oplus B$. Then $\operatorname{rank}\left(C_{H}\right)=\operatorname{rank}\left(C_{B}\right)+1$ and $\operatorname{ker}\left(C_{H}\right)=$ $\operatorname{ker}\left(C_{B}\right)+1$.

Proof: It follows directly from Lemma 2.1 and the fact that $C_{S_{q}}$ is a linear code of dimension 2 , which means that $\operatorname{rank}\left(C_{S_{q}}\right)=\operatorname{ker}\left(C_{S_{q}}\right)=2$.

Proposition 2.3: Let $H_{1}$ and $H_{2}$ be two GH matrices over $\mathbb{F}_{q}$ with $H=H_{1} \oplus H_{2}$. Let $K$ be a subfield of $\mathbb{F}_{q}$. If $C_{H_{1}}$ and $C_{H_{2}}$ are $K$-additive, then $C_{H}$ is also $K$-additive and $\operatorname{dim}_{K}\left(C_{H}\right)=\operatorname{dim}_{K}\left(C_{H_{1}}\right)+\operatorname{dim}_{K}\left(C_{H_{2}}\right)-1$.

Proof: It is straightforward using the same argument as in the proof of Lemma 2.1.

Lemma 2.4: Let $H=H_{1} \oplus\left[B_{1}, B_{2}, \ldots, B_{n}\right]$, where $H_{1}(q, \lambda)$ is a $\mathrm{GH}$ matrix over $\mathbb{F}_{q}$ and $B_{1}, B_{2}, \ldots, B_{n}$ are GH matrices over $\mathbb{F}_{q}$, where $n=q \lambda$. Then $\operatorname{rank}\left(C_{H}\right)=$ $\operatorname{rank}\left(C_{H_{1}}\right)+\operatorname{dim}\left(\left\langle C_{B_{1}} \cup C_{B_{2}} \cup \cdots \cup C_{B_{n}}\right\rangle\right)-1$.

Proof: The row vectors in a $\mathrm{GH}$ matrix are linearly generated by some of its rows. Let $\mathbf{v}_{i}$ be the $i$ th generator row of $H_{1}$. Using the same argument as in the proof of Lemma 2.1, we see that the vectors $\mathbf{v}_{i} \oplus \mathbf{0}$ and $\mathbf{0} \oplus \mathbf{w}_{j k}$ linearly generate $H$, where $\mathbf{w}_{j k}$ is the $j$ th generator row of $B_{k}$. Hence, we can conclude that $\operatorname{rank}\left(F_{H}\right)=\operatorname{rank}\left(F_{H_{1}}\right)+$ $\operatorname{dim}\left(\left\langle F_{B_{1}} \cup F_{B_{2}} \cup \cdots \cup F_{B_{n}}\right\rangle\right)$. Finally, by Lemma 1.1, $\operatorname{rank}\left(C_{H}\right)=1+\operatorname{rank}\left(F_{H}\right)=1+\operatorname{rank}\left(F_{H_{1}}\right)+\operatorname{dim}\left(\left\langle F_{B_{1}} \cup\right.\right.$ $\left.\left.F_{B_{2}} \cup \cdots \cup F_{B_{n}}\right\rangle\right)=\operatorname{rank}\left(C_{H_{1}}\right)+\operatorname{dim}\left(\left\langle C_{B_{1}} \cup C_{B_{2}} \cup \cdots \cup\right.\right.$ $\left.\left.C_{B_{n}}\right\rangle\right)-1$.

Corollary 2.5: Let $H=S_{q} \oplus\left[B_{1}, B_{2}, \ldots, B_{q}\right]$, where $B_{1}, B_{2}, \ldots, B_{q}$ are $\mathrm{GH}$ matrices over $\mathbb{F}_{q}$. Then $\operatorname{rank}\left(C_{H}\right)=$ $\operatorname{dim}\left(\left\langle C_{B_{1}} \cup C_{B_{2}} \cup \cdots \cup C_{B_{q}}\right\rangle\right)+1$.

Proof: It is straightforward from Lemma 2.4 and the fact that $C_{S_{q}}$ is a linear code of dimension 2 .

Lemma 2.6: Let $H=S_{q} \oplus\left[B_{1}, B_{2}, \ldots, B_{q}\right]$, where $B_{1}, B_{2}, \ldots, B_{q}$ are $\mathrm{GH}$ matrices over $\mathbb{F}_{q}$. If there exists, at least, one matrix $B_{i}$ which is not translation equivalent to any other $B_{j}$ for $i, j \in\{1,2, \ldots, q\}$ and $i \neq j$, then $\mathcal{K}\left(C_{H}\right)=\left\{\mathbf{0} \oplus \mathbf{x}=(\mathbf{x}, \mathbf{x}, \ldots, \mathbf{x}): \mathbf{x} \in \mathcal{K}\left(C_{B_{1}}\right) \cap \mathcal{K}\left(C_{B_{2}}\right) \cap\right.$ $\left.\cdots \cap \mathcal{K}\left(C_{B_{q}}\right)\right\}$.

Proof: Note that the row vectors in $S_{q}$ are linearly generated by one of its rows, for instance e. Hence, the rows in $S_{q}$ can be described by $\mathbf{e}_{i}=\omega^{i} \mathbf{e}$, where $\omega$ is a primitive element in $\mathbb{F}_{q}$ and $i \in\{1,2, \ldots, q-1\}$, and $\mathbf{e}_{q}=$ $\mathbf{0}$. Let $\mathbf{b}_{j k}$ be the $j$ th row of $B_{k}$. If $\mathbf{e}_{k} \oplus \mathbf{b}_{j k} \in \mathcal{K}\left(F_{H}\right)$, then adding $\mathbf{e}_{i} \oplus B_{i}$ we obtain elements belonging to $C_{H}$ for all $i \in\{1,2, \ldots, q\}$. Hence, $\mathbf{b}_{j k}+B_{i}=B_{s}$ for $i \in$ 
$\{1,2, \ldots, q\}$, where $\mathbf{e}_{s}=\mathbf{e}_{k}+\mathbf{e}_{i}$. Therefore, assuming the hypothesis in the statement, that is, $B_{i}$ is not translation equivalent to any other $B_{s}$ for $s \neq i$, we can conclude that when $\mathbf{e}_{k} \oplus \mathbf{b}_{j k} \in \mathcal{K}\left(F_{H}\right)$ we have that $\mathbf{e}_{k}=\mathbf{0}$ and $\mathbf{b}_{j q} \in \mathcal{K}\left(F_{B_{1}}\right) \cap \mathcal{K}\left(F_{B_{2}}\right) \cap \cdots \cap \mathcal{K}\left(F_{B_{q}}\right)$.

Finally, as the all-one vector $\mathbf{1}$ belongs to all the kernels $\mathcal{K}\left(C_{B_{i}}\right)$, for $i \in\{1,2, \ldots, q\}$, and also to $\mathcal{K}\left(C_{H}\right)$, we obtain the statement.

\section{KERNEL DIMENSION OF GH CODES}

In [16], it was proved that the Hadamard codes obtained from Hadamard matrices $H\left(2,2^{t-1}\right)$ over $\mathbb{F}_{2}$, with $t \geq 4$, have kernels of dimension $k \in\{1,2, \ldots, t-1, t+1\}$, and a construction of binary Hadamard codes of length $n=$ $2^{t}$, with $t \geq 4$, for each one of these values was given. In [17], this result was generalized to Hadamard matrices $H\left(2,2^{t-1} s\right)$ over $\mathbb{F}_{2}$ showing that if there exists a binary Hadamard code of length $4 s, s \neq 1$ odd, then there exist binary Hadamard codes of length $n=2^{t} s$ for all $t \geq 2$, with kernel of dimension $k$ for all $k \in\{1,2, \ldots t-1\}$. In this section, we include some results about the dimension of the kernel of $\mathrm{GH}$ codes with $q \neq 2$.

Proposition 3.1: Let $H(q, \lambda)$ be a $\mathrm{GH}$ matrix over $\mathbb{F}_{q}$, where $q=p^{e}$ and $p$ prime. Let $n=q \lambda=p^{t} s$ such that $\operatorname{gcd}(p, s)=1$. Then $1 \leq \operatorname{ker}\left(C_{H}\right) \leq \operatorname{ker}_{p}\left(C_{H}\right) \leq 1+t / e$.

Proof: Since $\mathcal{K}_{p}\left(F_{H}\right)$ is an $\mathbb{F}_{p}$-additive subcode of $F_{H}$, we have that $\left|\mathcal{K}_{p}\left(F_{H}\right)\right|=p^{d}$ for an integer $d$. Moreover, by the properties of the kernel, since $F_{H}$ can be written as the union of cosets of $\mathcal{K}_{p}\left(F_{H}\right)$, we have that $p^{d} \mid p^{t} s$. Then, $d \leq t$ since $\operatorname{gcd}(p, s)=1$. Therefore, $\operatorname{ker}_{p}\left(F_{H}\right)=d / e \leq$ $t / e$, which proves that $0 \leq \operatorname{ker}\left(F_{H}\right) \leq \operatorname{ker}_{p}\left(F_{H}\right) \leq t / e$. By Lemma 1.1, the result follows.

Proposition 3.2: Let $H(4, \lambda)$ be a GH matrix with $\lambda$ odd. Then $1 \leq \operatorname{ker}\left(C_{H}\right) \leq \operatorname{ker}_{2}\left(C_{H}\right) \leq 2$.

Proof: Straigtforward from Proposition 3.1

Note that the upper bound in Proposition 3.2 for $\operatorname{ker}\left(C_{H}\right)$ and $\operatorname{ker}_{2}\left(C_{H}\right)$ are tight as we can see by taking the unique normalized GH matrix $H(4,1)=S_{4}$ given by the multiplicative table of $\mathbb{F}_{4}$. In this case $\operatorname{ker}\left(C_{H}\right)=$ $\operatorname{ker}_{2}\left(C_{H}\right)=2$. The lower bound is also tight since the unique normalized $\mathrm{GH}$ matrix $H(4,3)$ has $\operatorname{ker}\left(C_{H}\right)=$ $\operatorname{ker}_{2}\left(C_{H}\right)=1$.

Lemma 3.3: For $q>3$, there exists at least two versions of $S_{q}$ which are not translation equivalent. Moreover, they have a trivial intersection.

Proof: Recall that $S_{q}$ is the matrix given by the multiplicative table of $\mathbb{F}_{q}$ and let $S_{q}^{\prime}$ be the matrix $S_{q}$ after a transposition of the second and third column. The entries of both matrices are elements from $\left\{0,1, \omega, \ldots, \omega^{q-2}\right\}$, where $\omega$ is a primitive element in $\mathbb{F}_{q}$. Both matrices $S_{q}$ and $S_{q}^{\prime}$ are $\mathrm{GH}$ matrices, but they are not translation equivalent. Indeed, $S_{q} \neq S_{q}^{\prime}$ and if there exists a vector $\mathbf{v}=\left(v_{1}, v_{2}, \ldots, v_{q}\right)$ such that $\mathbf{v}+S_{q}=S_{q}^{\prime}$, then $\mathbf{v} \in S_{q}^{\prime}$. Since $F_{S_{q}^{\prime}}$ is a linear code over $\mathbb{F}_{q},-\mathbf{v} \in S_{q}^{\prime}$. Let $\mathbf{w}$ be the row vector in $S_{q}$ that coincides with $\mathbf{- v}$ in the last $q-3$ coordinates. Note that $\mathbf{w}$ is completely determined by $\mathbf{v}$, since $q>3$. Then, we have that $\mathbf{v}+\mathbf{w}=\left(0, v_{2}-v_{3}, v_{3}-\right.$ $\left.v_{2}, 0, \ldots, 0\right)=\mathbf{0} \in S_{q}^{\prime}$, so $v_{2}=v_{3}$ and $\mathbf{v}=\mathbf{0}$.

Let $\mathbf{0}, \mathbf{1}, \boldsymbol{\omega}^{(1)}, \ldots, \boldsymbol{\omega}^{(q-2)}$ be the elements $0,1, \omega, \ldots, \omega^{q-2}$ repeated $\lambda$ times, respectively, where $\omega$ is a primite element in $\mathbb{F}_{q}$.

Proposition 3.4: For $q>2$, there exists a $\mathrm{GH}$ matrix $H(q, q)$ over $\mathbb{F}_{q}$ such that the $\mathrm{GH}$ code $C_{H}$ of length $n=q^{2}$ over $\mathbb{F}_{q}$ has $\operatorname{ker}\left(C_{H}\right)=2$.

Proof: A GH matrix $H(q, q)$ over $\mathbb{F}_{q}$ such that $\operatorname{ker}\left(C_{H}\right)=2$ can be obtained by using a switching construction. This kind of construction has already been used for Hadamard matrices over $\mathbb{F}_{2}$ in [16], [17]. Let $K$ be the linear subcode of $S^{2}=S_{q} \oplus S_{q}$ generated by the $q+1$ row vector $\mathbf{v}$, that is, $K=\langle\mathbf{v}\rangle$, where $\mathbf{v}=\left(\mathbf{0}, \mathbf{1}, \boldsymbol{\omega}^{(1)}, \ldots, \boldsymbol{\omega}^{(q-2)}\right)$. Then, we take a coset $K+$ $\mathbf{x} \subset S^{2}$ such that $\mathbf{x} \in S^{2} \backslash K$. Finally, we construct a matrix $H$ as $H=S^{2} \backslash(K+\mathbf{x}) \cup(K+\mathbf{x}+\mathbf{e})$, where $\mathbf{e}=(0, \ldots, 0,1, \ldots, 1,0, \ldots, 0)$ and the ones in $\mathbf{e}$ cover the positions from $q+1$ to $2 q$. Clearly, $F_{H}$ is nonlinear and $K \subseteq \mathcal{K}\left(F_{H}\right)$, so $K=\mathcal{K}\left(F_{H}\right)$ and $\operatorname{ker}\left(C_{H}\right)=2$ by Lemma 1.1.

To prove that $H$ is a GH matrix, we just need to show that the multisets $\left\{\mathbf{v}_{s}-\mathbf{w}_{s}: 1 \leq s \leq q^{2}\right\}$, for $\mathbf{w} \in S^{2} \backslash(K+$ $\mathbf{x}$ ) and $\mathbf{v} \in K+\mathbf{x}+\mathbf{e}$, contains every element of $\mathbb{F}_{q}$ exactly $q$ times. We have that $\mathbf{w}=\mathbf{k}_{1}+\mathbf{x}^{\prime}$ and $\mathbf{v}=\mathbf{k}_{2}+\mathbf{x}+\mathbf{e}$ for some $\mathbf{k}_{1}, \mathbf{k}_{2} \in K$ and $\mathbf{x}^{\prime} \notin K+\mathbf{x}$, so $\mathbf{v}-\mathbf{w}=\mathbf{k}^{\prime \prime}+\mathbf{x}^{\prime \prime}+\mathbf{e}$, where $\mathbf{k}^{\prime \prime} \in K$ and $\mathbf{x}^{\prime \prime}=\mathbf{x}-\mathbf{x}^{\prime} \in S^{2} \backslash K$. It is clear that $\mathbf{k}^{\prime \prime}+\mathbf{x}^{\prime \prime} \in S^{2}$ and each element of $\mathbb{F}_{q}$ appears once in each block of $q$ coordinates. Adding $\mathbf{e}$ to $\mathbf{k}^{\prime \prime}+\mathbf{x}^{\prime \prime}$, we just change the order of the elements in the second block of $q$ coordinates, so $H$ fulfills the condition to be a GH matrix.

Example 3.5: There are exactly two GH matrices $H(3,3)$ over $\mathbb{F}_{3}$, up to equivalence [11]. One of them is the Sylvester GH matrix $S^{2}$ which has $\operatorname{ker}\left(C_{S^{2}}\right)=3$. The other one can be constructed using the switching construction given by the proof of Proposition 3.4. That is, first we consider the linear subcode $K=\langle\mathbf{v}\rangle \subset S^{2}$ over $\mathbb{F}_{3}$, where $\mathbf{v}=(0,0,0,1,1,1,2,2,2)$. Then, we take for example the coset $K+\mathbf{x} \neq K$, where $\mathbf{x}=(0,1,2,0,1,2,0,1,2)$. Finally, the matrix $H=S^{2} \backslash(K+\mathbf{x}) \cup(K+\mathbf{x}+\mathbf{e})$, where $\mathbf{e}=(0,0,0,1,1,1,0,0,0)$, which is the matrix $H$ given in Equation (1), is a $\mathrm{GH}$ matrix such that the $\mathrm{GH}$ code $C_{H}$ of length $n=9$ has $\mathcal{K}\left(C_{H}\right)=\langle\mathbf{1}, \mathbf{v}\rangle$, so $\operatorname{ker}\left(C_{H}\right)=2$. Note that the rows in boldface in Equation (1) indicate those that are in $K+\mathbf{x}+\mathbf{e}$.

$$
H=\left(\begin{array}{lllllllll}
0 & 0 & 0 & 0 & 0 & 0 & 0 & 0 & 0 \\
\mathbf{0} & \mathbf{1} & \mathbf{2} & \mathbf{1} & \mathbf{2} & \mathbf{0} & \mathbf{0} & \mathbf{1} & \mathbf{2} \\
0 & 2 & 1 & 0 & 2 & 1 & 0 & 2 & 1 \\
0 & 0 & 0 & 1 & 1 & 1 & 2 & 2 & 2 \\
\mathbf{0} & \mathbf{1} & \mathbf{2} & \mathbf{2} & \mathbf{0} & \mathbf{1} & \mathbf{2} & \mathbf{0} & \mathbf{1} \\
0 & 2 & 1 & 1 & 0 & 2 & 2 & 1 & 0 \\
0 & 0 & 0 & 2 & 2 & 2 & 1 & 1 & 1 \\
\mathbf{0} & \mathbf{1} & \mathbf{2} & \mathbf{0} & \mathbf{1} & \mathbf{2} & \mathbf{1} & \mathbf{2} & \mathbf{0} \\
0 & 2 & 1 & 2 & 1 & 0 & 1 & 0 & 2
\end{array}\right)
$$

Theorem 3.6: For $q>2$, and any $h \geq 2$, except for $q=3$ and $h=2$, there exists a $\mathrm{GH}$ code $C_{H}$ of length 
$n=q^{h}$ over $\mathbb{F}_{q}$ with $\operatorname{ker}\left(C_{H}\right)=k$ if and only if $k \in$ $\{1,2, \ldots, h+1\}$. For $q=3$ and $h=2$, there exists a GH code $C_{H}$ of length $n=9$ over $\mathbb{F}_{3}$ with $\operatorname{ker}\left(C_{H}\right)=k$ if and only if $k \in\{2,3\}$.

Proof: By Proposition 3.1, any GH code $C_{H}$ of length $n=q^{h}$ over $\mathbb{F}_{q}$ has $\operatorname{ker}\left(C_{H}\right) \in\{1,2, \ldots, t / e+1\}=$ $\{1,2, \ldots, h+1\}$, since $h=t / e$.

For $q=3$ and $t=h=2$, there are exactly two nonequivalent GH matrices $H(3,3)$ over $\mathbb{F}_{3}$ [11]. Both such matrices are also described in Example 3.5, where it is shown that their corresponding GH codes have kernels of dimension 2 and 3 , respectively. Therefore, there exists a GH code $C_{H}$ of length $n=9$ over $\mathbb{F}_{3}$ with $\operatorname{ker}\left(C_{H}\right)=k$ if and only if $k \in\{2,3\}$. Let $H_{2}$ and $H_{3}$ be the GH matrices $H(3,3)$ such that $\operatorname{ker}\left(C_{H_{2}}\right)=2$ and $\operatorname{ker}\left(C_{H_{3}}\right)=3$. Note that $\mathrm{H}_{2}$ can be constructed from $\mathrm{H}_{3}$ using a switching construction, so $\mathcal{K}\left(C_{H_{2}}\right) \subseteq \mathcal{K}\left(C_{H_{3}}\right)=C_{H_{3}}$.

For $q=3$ and $h=3$, using the Kronecker sum construction, we can obtain two GH matrices $H\left(3,3^{2}\right)$ as $S_{q} \oplus H_{2}$ and $S_{q} \oplus H_{3}$ with kernel of dimension 3 and 4, respectively, by Corollary 2.2. By Lemma 2.6, the GH matrix $H\left(3,3^{2}\right)=S_{q} \oplus\left[H_{2}, H_{3}, \ldots, H_{3}\right]$ has $\operatorname{ker}\left(C_{H}\right)=2$. Again by Lemma 2.6, the GH matrix $H\left(3,3^{2}\right)=S_{q} \oplus\left[\pi\left(H_{2}\right), H_{3}, \ldots, H_{3}\right]$ has $\operatorname{ker}\left(C_{H}\right)=1$, where $\pi$ is a permutation such that $\mathcal{K}\left(C_{H_{2}}\right) \cap \mathcal{K}\left(C_{\pi\left(H_{2}\right)}\right)=$ $\langle\mathbf{1}\rangle$.

For $q>3$ and $h=2$, using the Kronecker sum construction, we can obtain GH matrices $H(q, q)=S^{2}=S_{q} \oplus S_{q}$ with $\operatorname{ker}\left(C_{H}\right)=3$ by Corollary 2.2 , and $H(q, q)=S_{q} \oplus$ $\left[S_{q}^{\prime}, S_{q}, \ldots, S_{q}\right]$, where $S_{q}^{\prime} \cap S_{q}=\{\mathbf{0}\}$, with $\operatorname{ker}\left(C_{H}\right)=1$ by Lemmas 2.6 and 3.3. By Proposition 3.4, we can also obtain a GH matrix $H(q, q)$ such that $\operatorname{ker}\left(C_{H}\right)=2$ using a switching construction.

We have seen that the statement is true for any $q>3$ and $h=2$; and for $q=3$ and $h \in\{2,3\}$. Finally, by induction we will prove the result for any $h \geq 2$. Suppose it is true for $n=q^{h-1}$, that is, there exists a GH code $C_{H_{i}}$ of length $q^{h-1}$ with kernel of dimension $i$ for all $i \in$ $\{1, \ldots, h\}$. By Corollary 2.2 , the GH code obtained from $S_{q} \oplus H_{i}$ has a kernel of dimension $i+1$. Note that $H_{1}$ and $H_{j}$ for any $j, j \neq 1$, have different ranks, which means that they are not translation equivalent. By Lemma 2.6, the GH code corresponding to $S_{q} \oplus\left[H_{1}, H_{j}, \ldots, H_{j}\right]$ has a kernel of dimension 1.

Lemma 3. 7: Let $C_{H}$ be a GH code of length $n=q^{h} s$ over $\mathbb{F}_{q}$, where $s \neq 1$ and $s$ is not a multiple of $q$. If $\operatorname{ker}\left(C_{H}\right)>1$, then $h \geq 2$.

Proof: If $\operatorname{ker}\left(C_{H}\right)>1$, then $\operatorname{ker}\left(F_{H}\right) \geq 1$ by Lemma 1.1. We can assume without loss of generality that $\mathbf{v}=\left(\mathbf{0}, \mathbf{1}, \boldsymbol{\omega}^{(1)}, \ldots, \boldsymbol{\omega}^{(q-2)}\right) \in \mathcal{K}\left(F_{H}\right)$. We consider all $n=q^{h} s$ coordinate positions divided into $q$ blocks of size $\lambda=q^{h-1} s$, such that the coordinates of $\mathbf{v}$ in each block coincide.

Let $\mathbf{x} \in F_{H} \backslash\langle\mathbf{v}\rangle$. Let $\eta_{\beta}^{(i)}$ be the number of times an element $\beta \in \mathbb{F}_{q}$ appears in the coordinates of the $i$ th block of $\mathbf{x}$. Since $\mathbf{x} \in F_{H}$, we have that

$$
\eta_{\alpha}^{(1)}+\eta_{\alpha}^{(2)}+\cdots+\eta_{\alpha}^{(q)}=\lambda
$$

for all $\alpha \in \mathbb{F}_{q}$. Adding these $q$ equations, we obtain

$$
\sum_{\alpha \in \mathbb{F}_{q}} \sum_{i=1}^{q} \eta_{\alpha}^{(i)} \equiv 0 \quad(\bmod q) .
$$

Since $\mathbf{x}+\omega^{j} \mathbf{v} \in F_{H}$, we have that

$$
\eta_{\alpha}^{(1)}+\eta_{\alpha-\omega^{j}}^{(2)}+\eta_{\alpha-\omega^{j+1}}^{(3)}+\cdots+\eta_{\alpha-\omega^{j+q-2}}^{(q)}=\lambda
$$

for all $\alpha \in \mathbb{F}_{q}$ and $j \in\{0, \ldots, q-2\}$. For a fixed $j \in$ $\{0, \ldots, q-2\}$, adding the $q$ equations, we obtain

$$
\begin{aligned}
\sum_{\alpha \in \mathbb{F}_{q}}\left(\eta_{\alpha}^{(1)}+\eta_{\alpha-\omega^{j}}^{(2)}\right. & \left.+\eta_{\alpha-\omega^{j+1}}^{(3)}+\cdots+\eta_{\alpha-\omega^{j+q-2}}^{(q)}\right) \\
& =\sum_{\alpha \in \mathbb{F}_{q}} \sum_{i=1}^{q} \eta_{\alpha}^{(i)} \equiv 0 \quad(\bmod q) .
\end{aligned}
$$

The size of each block is $\lambda$, so we also have that

$$
\sum_{\alpha \in \mathbb{F}_{q}} \eta_{\alpha}^{(i)}=\lambda
$$

for all $i \in\{1, \ldots, q\}$, and adding these $q$ equations, we obtain the same relation as in Equation (3). From these $q(q+1)$ equations, given by Equations (2), (4), and (5), we have seen that there are $q$ which are linear dependent, and the rest are linear independent. The fact that they are linear independent is easily seen by writing the equations in matrix form. The system of equations has a unique solution $\eta_{\alpha}^{(i)}=\lambda / q$ for all $\alpha \in \mathbb{F}_{q}$ and $i \in\{1, \ldots, q\}$, which means that $\lambda$ has to be a multiple of $q$, so $h \geq 2$.

Lemma 3.8: Let $C_{H}$ be a GH code of length $n=q^{h} s$ over $\mathbb{F}_{p}$, where $s \neq 1$ and $s$ is not a multiple of $q$. Then $\operatorname{ker}\left(C_{H}\right) \leq h$.

Proof: If $C_{H}$ has length $n=q s$, that is, if $h=1$, then $\operatorname{ker}\left(C_{H}\right)=1$ and the statement is true by Lemma 3.7. If $h>1$, assume that $C_{H}$ of length $n=q^{h} s$ has $\operatorname{ker}\left(C_{H}\right)=k>1$. Let $\mathbf{v} \in \mathcal{K}\left(C_{H}\right) \backslash\langle\mathbf{1}\rangle$. Let $I$ be the set of coordinates where the vector $\mathbf{v}$ is equal to one. We puncture $C_{H}$ eliminating all coordinates outside $I$. There are exactly $q$ copies of each vector, since for any $\mathbf{x} \in C_{H}$, the vector $\mathbf{x}+\boldsymbol{\omega}^{(j)}-\boldsymbol{\omega}^{(j)} \mathbf{v}$ is the same as $\mathbf{x}$ in the coordinates of $I$, for all $j \in\{0, \ldots, q-2\}$. Note that $\mathbf{x}+\boldsymbol{\omega}^{(j)}-\boldsymbol{\omega}^{(j)} \mathbf{v} \in C_{H}$, since $\boldsymbol{\omega}^{(j)}-\boldsymbol{\omega}^{(j)} \mathbf{v} \in \mathcal{K}\left(C_{H}\right)$. Let $C$ be the new code without repeated vectors. Note that $C$ has length $n=q^{h-1} s$ and a kernel of dimension $k-1$, since the independent vectors in $\mathcal{K}\left(C_{H}\right)$ are still independent in $\mathcal{K}(C)$ except $\mathbf{v}$ which coincides with 1 . Using the same argument as in the proof of Lemma 3.7, it is easy to see that $C$ is a $\mathrm{GH}$ code. By using the induction hypothesis $k-1 \leq h-1$, so $k \leq h$.

Theorem 3.9: Let $q=p, p$ prime, $s \neq 1$ such that $s$ is not a multiple of $q$, and $h \geq 2$. If there exists a GH matrix $H(q, s)$, then there exist GH codes $C_{H}$ of length $n=q^{h} s$ over $\mathbb{F}_{q}$ with $\operatorname{ker}\left(C_{H}\right)=k$ if and only if $k \in\{1,2, \ldots, h\}$.

Proof: If there exists a GH matrix $H(q, s)$, then the corresponding GH code $C_{H}$ of length $n=q s$ has $\operatorname{ker}\left(C_{H}\right)=1$, by Lemma 3.7 and Proposition 3.1. The upper bound for the dimension of the kernel is given by Lemma 3.8. Following the same argument as in the last 
part of the proof of Theorem 3.6, we have the statement.

\section{RANK OF GH CODES}

In [16], it was proved that the Hadamard codes obtained from Hadamard matrices $H\left(2,2^{t-1}\right)$ over $\mathbb{F}_{2}$, with $t \geq 3$, have ranks $r \in\{t+1, \ldots, n / 2\}$, and a construction of binary Hadamard codes of length $n=2^{t}$ for each one of these values was given. In [17], it was shown that if there exists a binary Hadamard code of length $4 s, s \neq 1$ odd, which always has rank $n-1$ [1], then there exist binary Hadamard codes of length $n=2^{t} s$ for all $t \geq 3$, with rank $r$ for all $r \in\{4 s+t-3, \ldots, n / 2\}$. For generalized Hadamard matrices $H\left(q, q^{h-1}\right)$, the lower bound $\operatorname{rank}\left(C_{H}\right) \geq h$ was shown in [21], where it was proved that a matrix of minimum rank is unique up to equivalence.

In this section, we give an upper bound for the rank proving that any GH matrix $H(q, \lambda)$ over $\mathbb{F}_{q}$ with $q>2$ is self-orthogonal, except $q=3$ and $\operatorname{gcd}(\lambda, 3)=1$. Moreover, for some particular cases, we specify lower and upper bounds on the rank, once the dimension of the kernel is given. Finally, GH codes having all different ranks between these bounds are constructed for some of these cases.

For vectors over $\mathbb{F}_{q}, q=p^{e}$ and $p$ prime, we have the $E u$ clidean inner product. Namely $[\mathbf{v}, \mathbf{w}]=\sum_{i=1}^{n} v_{i} w_{i}$ for any $\mathbf{v}, \mathbf{w} \in \mathbb{F}_{q}^{n}$. If $C$ is a code over $\mathbb{F}_{q}$ of length $n$, then we define the Euclidean orthogonal code as $C^{\perp}=\{\mathbf{v}:[\mathbf{v}, \mathbf{w}]=0$ for all $\mathbf{w} \in C\}$. Note that $C^{\perp}$ is always linear over $\mathbb{F}_{q}$ whether $C$ is or not. In fact, if $C$ is a nonlinear code, then $C^{\perp}=\langle C\rangle^{\perp}$. Moreover, we say that $C$ is Euclidean selforthogonal if $C \subseteq C^{\perp}$ and Euclidean self-dual if $C=C^{\perp}$.

Lemma 4.1: Let $H(q, \lambda)$ be a $\mathrm{GH}$ matrix over $\mathbb{F}_{q}$. Then $\mathbf{1} \in \mathcal{R}\left(F_{H}\right)^{\perp}$.

Proof: The sum of the elements of $\mathbb{F}_{q}, q=p^{e}$ and $p$ prime, is 0 . Indeed, the elements of the finite field $\mathbb{F}_{q}$ are the roots of the polynomial $x^{q}-x$ and the sum of all these roots is the coefficient of $x^{q-1}$, which is zero (except for $q=2)$. Then, since each row vector $\mathbf{v}$ in $H$ has all the elements in $\mathbb{F}_{q}$ repeated $\lambda$ times, we have, for $q \neq 2$, that $[\mathbf{1}, \mathbf{v}]=\lambda 0=0$ in $\mathbb{F}_{q}$ for all rows $\mathbf{v}$ in $H$. When $q=2$, since $\lambda$ is always even, we also obtain $[\mathbf{1}, \mathbf{v}]=0$ in $\mathbb{F}_{2}$.

Lemma 4.2: Let $H(q, \lambda)$ be a normalized $\mathrm{GH}$ matrix over $\mathbb{F}_{q}$. Then $(1,0, \ldots, 0) \in \mathcal{R}\left(F_{H}\right)^{\perp}$.

Proof: If the matrix is normalized, then the first coordinate is 0 and the result follows.

Proposition 4.3: Let $H(q, \lambda)$ be a normalized GH matrix over $\mathbb{F}_{q}$. Then $\operatorname{rank}\left(C_{H}\right) \leq n-1$, where $n=q \lambda$.

Proof: By the previous two lemmas, we have that the dimension of $\mathcal{R}\left(F_{H}\right)^{\perp}=F_{H}^{\perp}$ is at least 2. Then, $\operatorname{rank}\left(F_{H}\right)=n-\operatorname{rank}\left(F_{H}^{\perp}\right) \leq n-2$. By Lemma 1.1, $\operatorname{rank}\left(C_{H}\right) \leq n-1$.

The upper bound given by Proposition 4.3 can be met, for instance, for the $\mathrm{GH}$ matrices given in Examples 4.4 and 4.5 .

Example 4.4: The normalized Hadamard matrices $H(2,2 \mu)$ over $\mathbb{F}_{2}$ satisfy that $\operatorname{rank}\left(F_{H}\right)=4 \mu-2$, so $\operatorname{rank}\left(C_{H}\right)=4 \mu-1$, as long as $\mu$ is odd [1].
Example 4.5: The unique normalized GH matrix $S_{3}=$ $H(3,1)$ over $\mathbb{F}_{3}$, given by the multiplicative table of $\mathbb{F}_{3}$, has $\operatorname{rank}\left(C_{H}\right)=2$. Consider the normalized $\mathrm{GH}$ matrix $H(3,2)$ over $\mathbb{F}_{3}$, given by $(6)$. It is easy to verify that there is an unique normalized GH matrix $H(3,2)$ over $\mathbb{F}_{3}$, up to equivalence. In this case, $\operatorname{rank}\left(F_{H}\right)=4$, so $\operatorname{rank}\left(C_{H}\right)=5$.

$$
H(3,2)=\left(\begin{array}{llllll}
0 & 0 & 0 & 0 & 0 & 0 \\
0 & 0 & 1 & 1 & 2 & 2 \\
0 & 1 & 2 & 0 & 1 & 2 \\
0 & 1 & 0 & 2 & 2 & 1 \\
0 & 2 & 1 & 2 & 1 & 0 \\
0 & 2 & 2 & 1 & 0 & 1
\end{array}\right)
$$

There is also an unique normalized GH matrix $H(3,4)$ over $\mathbb{F}_{3}$, up to equivalence [4], which has $\operatorname{rank}\left(C_{H}\right)=11$.

Note that the GH matrices from the previous two examples generate codes that are not self-orthogonal. However, there are many others GH matrices such that they do lead to self-orthogonal codes. For example, it is known that the Hadamard matrices $H(2,2 \mu)$ over $\mathbb{F}_{2}$ generate selforthogonal codes when $\mu$ is even [1]. Moreover, in [8], it is shown by computer that the code generated by any $\mathrm{GH}$ matrix $H(4,4)$ over $\mathbb{F}_{4}$ is self-orthogonal.

What we do next is to show that any $\mathrm{GH}$ matrix $H(q, \lambda)$ over $\mathbb{F}_{q}$, with $q>3$, or $q=3$ and $\operatorname{gcd}(3, \lambda) \neq 1$, generates a self-orthogonal code.

Let $\mathbb{Z}_{2 p}$ be the ring of integers modulo $2 p$. A monic polynomial $f(x) \in \mathbb{Z}_{2 p}[x]$ is called monic basic irreducible (primitive) if computing it modulo 2 we obtain an irreducible (primitive) polinomial $\bar{f}(x) \in \mathbb{F}_{2}[x]$. We define the ring $R_{(2 p)^{e}}=\mathbb{Z}_{2 p}[x] /\langle f(x)\rangle$, where $f(x)$ is a monic basic irreducible polynomial of degree $e$ (which always exists). The ring $R_{(2 p)^{e}}$ has a maximal ideal $\langle p\rangle$ and its residue field $R_{(2 p)^{e}} /\langle p\rangle$ is isomorphic to the field $\mathbb{F}_{p^{e}}$ of order $p^{e}$. In the ring $R_{(2 p)^{e}}$ there exists a nonzero element $\omega$ of order $p^{e}-1$, which is a root of a basic primitive polynomial $f(x)$ of degree $e$ over $\mathbb{Z}_{2 p}$ and $R_{(2 p)^{e}}=\mathbb{Z}_{2 p}[\omega]$. Let $T=\left\{0,1, \omega, \ldots, \omega^{p^{e}-2}\right\}$, then any element of $R_{(2 p)^{e}}$ can be written uniquely, using a $p$-adic representation, as $a+p b$, where $a, b \in T$. Analogously, the elements in $\mathbb{F}_{p^{e}}$ are those in $\bar{T}=\left\{0,1, \bar{\omega}, \ldots, \bar{\omega}^{2^{e}-2}\right\}$, where $\bar{\omega}$ is a root of the primitive polynomial $\bar{f}(x) \in \mathbb{F}_{p}[x]$.

Lemma 4.6: If $p=2$ and $e>1$, then $\sum_{\alpha \in T} \alpha^{2} \equiv 0$ $(\bmod 2)$.

Proof: Since $\operatorname{gcd}\left(2,2^{e}-1\right)=1$, every element of the multiplicative cyclic group $\langle\omega\rangle$ can be written as a square element of $\langle\omega\rangle$. Hence, $\sum_{\alpha \in T} \alpha^{2}=\sum_{\alpha \in T} \alpha$. Taking modulo 2 and noting that the sum of all elements in $\bar{T}$ is zero (indeed, the elements in $\bar{T}$ are the roots of $x^{2^{e}-1}-x \in$ $Z_{2}[x]$ and the addition of all of them is the coefficient of $x^{2^{e}-2}$, which is zero for $e \geq 2$ ) we obtain, using the 2-adic presentation, $\sum_{\alpha \in T} \alpha=2 b$ with $b \in T$. Now, we show that $2 b=0$. Squaring each side the last equation $\sum_{\alpha \in T} \alpha=2 b$ we obtain $\sum_{\alpha \in T} \alpha^{2}+\sum_{i \neq j ; i, j \in\left\{0, \ldots, 2^{e}-2\right\}} \omega^{i} \omega^{j}=0$. Hence, $2 b+\sum_{k \in\left\{0, \ldots, 2^{e}-2\right\}} \lambda_{k} \omega^{k}=0$, where $\lambda_{k}$ stands for the number of ways we can split $k$ or $2^{e}-1+k$ in two different addends $i, j \in\left\{0, \ldots, 2^{e}-2\right\}$. It is easy to see 
that $\lambda_{k}=2^{e}-2$ for all $k \in\left\{0, \ldots, 2^{e}-2\right\}$ and so $2 b+\left(2^{e}-2\right) \sum_{\alpha \in T} \alpha=0$. Finally, $\left(2^{e}-1\right) 2 b=0$. Therefore $2 b=0$, which proves the statement.

Lemma 4.7: Let $p$ be an odd prime. If $p^{e}>3$, then $\sum_{x \in \mathbb{F}_{p^{e}}} x^{2} \equiv 0(\bmod 2 p)$. If $p^{e}=3$, then $\sum_{x \in \mathbb{F}_{3}} x^{2} \equiv 2$ $(\bmod 3)$.

Proof: For $p^{e}=3$ the result is straightforward. For $p^{e}>3$, let $a \in \mathbb{F}_{p^{e}}$ such that $a^{2} \neq 0,1$. As long as $p^{e}>3$ this is easily done. Then

$$
\sum_{x \in \mathbb{F}_{p} e} x^{2}=\sum_{x \in \mathbb{F}_{p^{e}}}(a x)^{2}=a^{2} \sum_{x \in \mathbb{F}_{p^{e}}} x^{2} .
$$

This gives that $\sum_{x \in \mathbb{F}_{p} e} x^{2}=0$ in $\mathbb{F}_{p^{e}}$.

Now split the nonzero elements of $\mathbb{F}_{p^{e}}$ into two disjoint sets $A$ and $B$ such that $x \in A$ if and only if $-x \in B$. Since $p \neq 2$, the elements $a$ and $-a$ are always distinct. Then $\sum_{x \in A} x^{2}=\sum_{x \in B} x^{2}$ and $\sum_{x \in \mathbb{F}_{p} e} x^{2}=$ $\sum_{x \in A} x^{2}+\sum_{x \in B} x^{2}$. This gives that $2 \sum_{x \in A} x^{2}=0$ and so $\sum_{x \in A} x^{2}=0$ in $\mathbb{F}_{p^{e}}$. Then we have that

$$
\sum_{x \in \mathbb{F}_{p} e^{2}} x^{2}=2 \sum_{x \in A} x^{2} \equiv 0 \quad(\bmod 2 p) .
$$

Let $\mathbf{v}, \mathbf{w} \in \mathbb{F}_{p^{e}}^{n}$. The Euclidian inner product $[\mathbf{v}, \mathbf{w}]=$ $\sum v_{i} w_{i}$ is computed in $\mathbb{F}_{p^{e}}$, but we can substitute the elements $\left\{0,1, \bar{\omega}, \ldots, \bar{\omega}^{p^{e}-2}\right\}$ of $\mathbb{F}_{p^{e}}$ by the corresponding representatives $\left\{0,1, \omega, \ldots, \omega^{p^{e}-2}\right\}$ in $R_{(2 p)^{e}}$ and we call $[\mathbf{v}, \mathbf{w}]_{2 p} \in R_{(2 p)^{e}}$ the result we obtain on computing the above inner product.

Lemma 4.8: Let $H\left(p^{e}, \lambda\right)$ be a $\mathrm{GH}$ matrix over $\mathbb{F}_{p^{e}}$. Let $\mathbf{v}$ and $\mathbf{w}$ be two rows of $H$. If $p^{e}>3$, then $[\mathbf{v}, \mathbf{v}]_{2 p}=0$, $[\mathbf{w}, \mathbf{w}]_{2 p}=0$ and $[\mathbf{v}+\mathbf{w}, \mathbf{v}+\mathbf{w}]_{2 p}=0$. If $p^{e}=3$, then $[\mathbf{v}, \mathbf{v}]=[\mathbf{w}, \mathbf{w}]=[\mathbf{v}+\mathbf{w}, \mathbf{v}+\mathbf{w}]=2 \lambda$.

Proof: We have that every element of $\mathbb{F}_{p^{e}}$ appears $\lambda$ times in $\mathbf{v}, \mathbf{w}$ and $\mathbf{v}+\mathbf{w}$. Then the result follows from Lemmas 4.6 and 4.7 .

We can now prove our desired result.

Theorem 4.9: Let $H\left(p^{e}, \lambda\right)$ be a GH matrix over $\mathbb{F}_{p^{e}}$, with $p^{e}>3$, or $p^{e}=3$ and $\lambda$ a multiple of 3 . Then $\mathcal{R}\left(F_{H}\right)$ is self-orthogonal.

Proof: Let $\mathbf{v}, \mathbf{w}$ be rows of $H$. For $p^{e}>3$ we know that $[\mathbf{v}, \mathbf{v}]_{2 p}=[\mathbf{v}+\mathbf{w}, \mathbf{v}+\mathbf{w}]_{2 p}=0$ by Lemma 4.8. Then, we have

$$
\begin{aligned}
0=[\mathbf{v}+\mathbf{w}, \mathbf{v}+\mathbf{w}]_{2 p} & =\sum\left(v_{i}+w_{i}\right)^{2} \\
& =\sum v_{i}{ }^{2}+w_{i}{ }^{2}+2 \sum v_{i} w_{i} \\
& =[\mathbf{v}, \mathbf{v}]_{2 p}+[\mathbf{w}, \mathbf{w}]_{2 p}+2 \sum v_{i} w_{i} \\
& =2 \sum v_{i} w_{i} .
\end{aligned}
$$

Since $0=2 \sum v_{i} w_{i}$ in $R_{(2 p)^{e}}$, we have that $\sum v_{i} w_{i} \equiv 0$ $(\bmod p)$. Hence $[\mathbf{v}, \mathbf{w}]=0$, which gives that $\mathcal{R}\left(F_{H}\right)$ is a self-orthogonal code.

For $p^{e}=3$ and $\lambda$ a multiple of 3 we have that

$$
\begin{aligned}
2 \lambda=[\mathbf{v}+\mathbf{w}, \mathbf{v}+\mathbf{w}] & =[\mathbf{v}, \mathbf{v}]+[\mathbf{w}, \mathbf{w}]+2 \sum v_{i} w_{i} \\
& =2 \lambda+2 \lambda+2 \sum v_{i} w_{i},
\end{aligned}
$$

so $2 \lambda \equiv 2 \sum v_{i} w_{i}(\bmod 3)$. If $\lambda$ is a multiple of 3 , we have $\sum v_{i} w_{i} \equiv 0(\bmod 3)$ and $[\mathbf{v}, \mathbf{w}]=0$, which gives that $\mathcal{R}\left(F_{H}\right)$ is a self-orthogonal code.

This leads to the following corollary.

Corollary 4.10: Let $H(q, \lambda)$ be a normalized GH matrix over $\mathbb{F}_{q}$, with $q>3$, or $q=3$ and $\lambda$ a multiple of 3 . Then $\operatorname{rank}\left(C_{H}\right) \leq\lfloor n / 2\rfloor$, where $n=q \lambda$.

Proof: By Theorem 4.9, we have that $\mathcal{R}\left(F_{H}\right)$ is selforthogonal. Moreover, by Lemmas 4.1 and 4.2 , we have that $(1,0, \ldots, 0)$ and $\mathbf{1}$ are in $\mathcal{R}\left(F_{H}\right)^{\perp}=F_{H}^{\perp}$ but not in $\mathcal{R}\left(F_{H}\right)$. Then $\operatorname{rank}\left(F_{H}^{\perp}\right) \geq 2+\operatorname{rank}\left(F_{H}\right)$ and $n-$ $\operatorname{rank}\left(F_{H}\right) \geq 2+\operatorname{rank}\left(F_{H}\right)$ which gives $\operatorname{rank}\left(F_{H}\right) \leq n / 2-1$. By Lemma 1.1, $\operatorname{rank}\left(C_{H}\right) \leq n / 2$.

Corollary 4.11: Let $H\left(q, q^{h-1}\right)$ be a GH matrix over $\mathbb{F}_{q}$, with $q>3$ and $h \geq 1$, or $q=3$ and $h \geq 2$. Then $\operatorname{rank}\left(C_{H}\right) \in\left\{h+1, \ldots,\left\lfloor q^{h} / 2\right\rfloor\right\}$.

Proof: For $q=3$ and $h \geq 2$, and for $q>3$ and $h \geq$ 1 , by Corollary 4.10 , we have that $\operatorname{rank}\left(C_{H}\right) \leq\lfloor n / 2\rfloor=$ $\left\lfloor q^{h} / 2\right\rfloor$. If $H=S^{h}$, where $S^{h}$ is the generalized Sylvester Hadamard matrix of order $q^{h}$, then $C_{H}$ is a linear code of length $n=q^{h}$ over $\mathbb{F}_{q}$, so $\operatorname{rank}\left(C_{H}\right)=h+1$ and the result follows.

Proposition 4.12: Let $H\left(q, q^{h-1}\right)$ be a GH matrix over $\mathbb{F}_{q}$, with $q>2$ and $h \geq 1$. Let $r=\operatorname{rank}\left(C_{H}\right)$ and $k=$ $\operatorname{ker}\left(C_{H}\right)$. Then,

(i) if $k=1$, then $h+2 \leq r \leq\left\lfloor q^{h} / 2\right\rfloor$;

(ii) if $2 \leq k \leq h$, then $h+2 \leq r \leq k+q^{h+1-k}-1$;

(iii) if $k=h+1$, then $r=h+1$.

Proof: First note that when $q=3$ and $h=1$, the $\mathrm{GH}$ matrix $H(3,1)$ is unique up to equivalence, and generates a linear code $C_{H}$, so $r=k=h+1=2$.

For all other cases, we can use the following argument. We know that $\mathcal{K}\left(C_{H}\right)$ is the largest linear subspace in $C_{H}$ such that $C_{H}$ can be written as the union of cosets of $\mathcal{K}\left(C_{H}\right)$. Since there are $q^{h+1-k}$ cosets in $C_{H}$, and $r$ is maximum when each coset contributes an independent vector, we have that $r \leq k+q^{h+1-k}-1$. This same argument was used in [16], [17] for binary Hadamard codes and in [20] for 1-perfect codes. If $k=1$, we have that $r \leq\left\lfloor q^{h} / 2\right\rfloor$, by Corollary 4.11, and because $q^{h} / 2<k+q^{h+1-k}-1=q^{h}$. Finally, if $k=h+1$, then $C_{H}$ is linear, so $r=h+1$. For the lower bound, just note that if $k \leq h$, then $C_{H}$ is nonlinear, so $r \geq h+2$.

The bounds given by Corollary 4.11 and Proposition 4.12 can be met, for example, for the $\mathrm{GH}$ matrices $H(4,4)$ over $\mathbb{F}_{4}$, as we can see in Example 4.13.

Example 4.13: It is known that there are exactly 226 nonequivalent $\mathrm{GH}$ matrices $H(4,4)$ over $\mathbb{F}_{4}$ having $\operatorname{rank}\left(C_{H}\right) \in\{3,4,5,6,7,8\}$ [8]. Moreover, the number of such $\mathrm{GH}$ matrices with respect to the pair $\left(\operatorname{rank}\left(C_{H}\right)\right.$, $\left.\operatorname{ker}\left(C_{H}\right)\right)$ is given by Table II. From this table, it is easy to see that the bounds on $\operatorname{rank}\left(C_{H}\right)$, once $\operatorname{ker}\left(C_{H}\right)$ is given, satisfy Proposition 4.12.

Corollary 4.14: Let $H\left(q, q^{h-1}\right)$ be a GH matrix over $\mathbb{F}_{q}$ with $q>2$ and $h \geq 1$. If $C_{H}$ is self-dual, then $q$ is even and $\operatorname{ker}\left(C_{H}\right)=1$. 
TABLE II

Parameters $\operatorname{rank}\left(C_{H}\right)$ And $\operatorname{ker}\left(C_{H}\right)$ Of GH matrices $H(4,4)$ OVER $\mathbb{F}_{4}$.

\begin{tabular}{r|cccccc} 
& \multicolumn{7}{|c}{$\operatorname{rank}\left(C_{H}\right)$} \\
$\operatorname{ker}\left(C_{H}\right)$ & 3 & 4 & 5 & 6 & 7 & 8 \\
\hline 3 & 1 & & & & & \\
2 & & 7 & 8 & & & \\
1 & & 3 & 92 & 55 & 57 & 3
\end{tabular}

Proof: If $C_{H}$ is self-dual, then $\operatorname{rank}\left(C_{H}\right)=n / 2$. Since $n / 2$ must be an integer, $q$ must be even. Therefore, the result follows from Proposition 4.12.

Proposition 4.15: For $q>2$, and any $h \geq 2$ such that $\lceil(h+2) / 2\rceil \leq k \leq h$, there exists a $\mathrm{GH}$ code $C_{H}$ of length $n=q^{h}$ over $\mathbb{F}_{q}$ with $\operatorname{ker}\left(C_{H}\right)=k$ and $\operatorname{rank}\left(C_{H}\right)=r$ if and only if $r \in\left\{h+2, \ldots, k+q^{h+1-k}-1\right\}$.

Proof: We will see that the corresponding GH matrices $H\left(q, q^{h-1}\right)$ can be generated by using a switching construction. Let $S^{h}$ be the Sylvester GH matrix $H\left(q, q^{h-1}\right)$. We can assume without loss of generality that $S^{h}$ is generated by the vectors $\mathbf{v}_{1}, \ldots, \mathbf{v}_{h}$ of length $n=q^{h}$, where

$\mathbf{v}_{i}=\left(\mathbf{0}_{i}, \mathbf{1}_{i}, \boldsymbol{\omega}_{i}^{(1)}, \ldots, \boldsymbol{\omega}_{i}^{(q-2)}, \ldots, \mathbf{0}_{i}, \mathbf{1}_{i}, \boldsymbol{\omega}_{i}^{(1)}, \ldots, \boldsymbol{\omega}_{i}^{(q-2)}\right)$,

$\mathbf{0}_{i}, \mathbf{1}_{i}, \boldsymbol{\omega}_{i}^{(1)}, \ldots, \boldsymbol{\omega}_{i}^{(q-2)}$ are the elements $0,1, \omega, \ldots, \omega^{q-2}$ repeated $q^{h-i}$ times, respectively, and $\omega$ is a primite element in $\mathbb{F}_{q}$, for all $i \in\{1, \ldots, h\}$. Let $K$ be the linear subcode of $S^{h}$ generated by the $k-1$ row vectors $\mathbf{v}_{1}, \ldots, \mathbf{v}_{k-1}$. Note that all $n=q^{h}$ coordinates are naturally divided into $q^{k-1}$ groups of size $q^{h-k+1}$, which will be referred to as blocks, such that the columns of $K$ in a block coincide.

The rows of $S^{h}$ can be partitioned into $q^{h-k+1}$ cosets of $K$. We can take any coset $K+\mathbf{x}_{1} \subset S^{h}$ such that $\mathbf{x}_{1} \in$ $S^{h} \backslash K$ and construct a matrix $H$ as $H=S^{h} \backslash\left(K+\mathbf{x}_{1}\right) \cup$ $\left(K+\mathbf{x}_{1}+\mathbf{e}_{1}\right)$, where $\mathbf{e}_{1}$ is the vector of length $n=q^{h}$ with ones in the positions given by the second block and zeros elsewhere. Clearly, $\operatorname{rank}\left(F_{H}\right)=h+1$ and $K \subseteq \mathcal{K}\left(F_{H}\right)$. Moreover, it is easy to prove that $K=\mathcal{K}\left(F_{H}\right)$. Therefore, $\operatorname{ker}\left(F_{H}\right)=k-1$, which gives $\operatorname{ker}\left(C_{H}\right)=k$ by Lemma 1.1.

This process can be performed several times. Let $\mathbf{e}_{s}$ be the vector of length $n=q^{h}$ with ones in the positions given by the $(s+1)$ th block and zeros elsewhere, for all $s \in\left\{1, \ldots, q^{h+1-k}+k-h-2\right\}$. Since $\lceil(h+2) / 2\rceil \leq k$, we have that $k-1 \geq h-k+1$. Therefore, $q^{k-1} \geq q^{h-k+1} \geq$ $q^{h+1-k}+k-h-2$, so there are enough blocks to define vector $e_{s}$. Let $\mathbf{x}_{1}, \mathbf{x}_{2}, \ldots, \mathbf{x}_{s} \in S^{h} \backslash K$ such that $\bigcap_{j=1}^{s}(K+$ $\left.\mathbf{x}_{j}\right)=\emptyset$. Then, we can construct the matrix

$$
H=\left(S^{h} \backslash \bigcup_{j=1}^{s}\left(K+\mathbf{x}_{j}\right)\right) \cup \bigcup_{j=1}^{s}\left(K+\mathbf{x}_{j}+\mathbf{e}_{j}\right) .
$$

Again, it is clear that $\operatorname{rank}\left(C_{H}\right)=h+1+s$ and $\operatorname{ker}\left(C_{H}\right)=$ $k$. To prove that $H$ is a $\mathrm{GH}$ matrix, we can use the same argument as in the proof of Proposition 3.4.

\section{Conclusions}

We have established lower and upper bounds for the dimension of the kernel and rank of codes constructed from
GH matrices over $\mathbb{F}_{q}$. For some cases, we proved that these bounds are tight, by constructing GH matrices for each possible rank, once the dimension of the kernel is given. Although ordinary Hadamard matrices are a specific case of GH matrices, in general, the results obtained in [16] and [17] on the bounds for the rank and dimension of kernel when $q=2$ can not be straightforwardly obtained from the results given in the current paper. Moreover, note that, the results where the kernel is involved for $q=2$ are not exactly the same as for any $q>2$, since it is not possible to obtain a nonlinear binary Hadamard code of length $n=2^{t}$ having a kernel of dimension $t$. When $q>2$, there are specific features in the proofs of the mentioned bounds, although, many techniques used in [16] and [17] have also been used in this paper.

Further research on this topic would include giving the construction of a GH matrix $H(q, \lambda)$ over $\mathbb{F}_{q}$ for each possible pair $\left(\operatorname{rank}\left(C_{H}\right), \operatorname{ker}\left(C_{H}\right)\right.$ ) or providing similar results for the parameters $p$-rank and $p$-kernel of these codes. Another direction of future research could be to focus on the rank and kernel of $\mathbb{F}_{p}$-additive GH codes, which are the ones having the $p$-rank equal to the $p$-kernel, or in a more general way, study $K$-additive GH codes, where $K$ is a subfield of $\mathbb{F}_{q}$.

\section{REFERENCES}

[1] E. F. Assmus and J. D. Key, Designs and Their Codes, Cambridge University Press, Great Britain, 1992.

[2] H. Bauer, B. Ganter, and F. Hergert, "Algebraic techniques for nonlinear codes," Combinatorica, vol. 3, pp. 21-33, 1983.

[3] G. Cohen, I. Honkala, S. Litsyn, and A. Lobstein, Covering Codes, North-Holland, Amsterdam, 1997.

[4] W. de Launey, "On the non-existance of generalized Hadamard matrices," Journal of Statistical Planning and Inference, vol. 10(3), pp. 385-396, 1984.

[5] C. Fernández-Córdoba, J. Pujol, and M. Villanueva, "On rank and kernel of $\mathbb{Z}_{4}$-linear codes," Lecture Notes in Computer Science, vol. 5228, pp. 46-55, 2008.

[6] C. Fernández-Córdoba, J. Pujol, and M. Villanueva, " $\mathbb{Z}_{2} \mathbb{Z}_{4-}$ linear codes: rank and kernel," Des. Codes Cryptography, vol. 56, pp. 43-59, 2010.

[7] A. R. Hammond, P. V. Kumar, A. R. Calderbank, N. J. A. Sloane, and P. Solé, "The $\mathbb{Z}_{4}$-linearity of kerdock, preparata, goethals and related codes," IEEE Trans. Inform. Theory, vol. 40, pp. 301-319, 1994.

[8] M. Harada, C. Lam, and V. Tonchev, "Symmetric $(4,4)$-nets and generalized Hadamard matrices over groups of order 4," Des. Codes Cryptography, vol. 34, pp. 71-87, 2005.

[9] D. Jungnickel, "On difference matrices, resolvable designs and generalized Hadamard matrices," Math. Z., vol. 167, pp. 49-60, 1979.

[10] F. I. MacWilliams and N. J. Sloane, The Theory of ErrorCorrecting Codes, North-Holland, New York, 1977.

[11] V. C. Mavron and V. D. Tonchev, "On symmetric nets and generalized Hadamard matrices from affine designs," Journal of Geometry, vol. 67, pp. 180-187, 2000.

[12] G. Nebe, E. M. Rains, and N. J. A. Sloane, "Self-Dual Codes and Invariant Theory," Algorithms and Computation in Mathematics, Berlin/Heidelberg: Springer-Verlag, vol. 17, 2006.

[13] Jong-Seon No and H.Y. Song, "Generalized Sylvester-type Hadamard matrices," IEEE International Symposium on Information Theory, June 2000, pp. 472.

[14] K. T. Phelps, M. LeVan, "Kernels of nonlinear Hamming codes," Des. Codes Cryptography, vol. 6, pp. 247-257, 1995.

[15] K. T. Phelps, J. Rifà, and M. Villanueva, "Kernels and $p$-kernels of $p^{r}$-ary 1-perfect codes," Des. Codes Cryptography, vol. 37, pp. 243-261, 2005. 
[16] K. T. Phelps, J. Rifà, and M. Villanueva, "Rank and kernel of binary Hadamard codes," IEEE Trans. Inform. Theory, vol. 51, no. 11, pp. 3931-3937, 2005.

[17] K. T. Phelps, J. Rifà, and M. Villanueva, "Hadamard codes of length $2^{t} s$ ( $s$ odd): Rank and kernel," Lecture Notes in Computer Science, vol. 3857, pp. 328-337, 2006.

[18] K. T. Phelps, J. Rifà, and M. Villanueva, "On the additive (Z4linear and non-Z4- linear) Hadamard codes: rank and kernel," IEEE Trans. Inform. Theory, vol. 52(1), pp. 316-319, 2006.

[19] K. T. Phelps and M. Villanueva, "Ranks of $q$-ary 1-perfect codes," Des. Codes Cryptography, vol. 27, pp. 139-144, 2002.

[20] K. T. Phelps and M. Villanueva, "On perfect codes: rank and kernel," Des. Codes Cryptography, vol. 27, pp. 183-194, 2002.

[21] V. D. Tonchev, "On generalized Hadamard matrices of minimum rank," Finite Fields Appl., vol. 10, pp. 522-529, 2004.

[22] S. S. Shrikhande, "Generalized Hadamard matrices and orthogonal arrays of strength two," Canad. J. Math., vol. 16, pp. 736$740,1964$.

Steven Dougherty received his Ph.D. in 1992 at Lehigh University. He is currently a full professor of Mathematics at the University of Scranton. He is the recipient of the 2004 Hasse prize given by the Mathematical Association of America. He has written papers with 49 coauthors and has lectured in 12 countries. His research interests are coding theory, combinatorics and abstract algebra.

Josep Rifà was born in Manlleu, Catalonia (Spain) in July 1951. He received the graduate degree in Sciences (Mathematical Section) in 1973, from the University of Barcelona and the Ph.D. degree in Sciences (Computer Sciences Section) in 1987, from the UAB (Autonomous University of Barcelona). Since 1974 he was an assistant professor in the Mathematics Department, Barcelona University. In 1987 he joined the UAB and since 1992 he has been a full professor in this University. He was the former Head of Information and Communications Engineering Department at UAB as well as the former Vice-chairman of the Spanish Chapter of Information Theory of IEEE. He has worked in several projects of Spanish CICYT and other organizations on subjects related to digital communications, error correcting codes and encryption of digital information. His research interests include information theory, coding theory and cryptography.

Mercè Villanueva was born in Roses, Catalonia, Spain, in January 1972. She received her Bachelor's degree in Mathematics in 1994 from the Autonomous University of Barcelona and her Ph.D. degree in Science (Computer Science Section) in 2001 from the same university. In 1994 she joined the Department of Information and Communications Engineering, at the Autonomous University of Barcelona, as an Assistant Professor, and was promoted to Associate Professor in 2002. Her research interests include subjects related to combinatorics, algebra, coding theory, and graph theory. 